examine age and gender differences in fall risk factors among middle-aged adults who presented to an emergency department (ED) due to a fall.

Methods A sample of patients aged 40-64 years who presented to one of the three chosen public hospital EDs due to an unintentional fall were approached for a survey between July 2013 and Mar 2014. Patients were eligible when a fall event was indicated in the Presenting Complaint Code or Presenting Problem text field in the Emergency Department Information System (EDIS) database. Information obtained from the survey included demographic data and other health information which was collected as potentially important predictors of falls. Potential fall predictors include a history of falls, having co-morbid conditions and medications, feeling of losing balance, hazardous drinking patterns, poor physical functioning, insufficient exercises, and being underweight or obese.

Results There were 305 participants presenting to one of the three EDs in South-East Queensland during the period. The majority were females $(59.3 \%)$ and the mean age of the sample was 54 years (standard deviation 7.2). Age and gender were associated with different fall risk factors among the middle-aged adults. In the multivariable analysis, increasing age was associated with poor physical functioning (odds ratio (OR) 1.05, 95\% CI: 1.003-1.09). Women were two times more likely than men to report a fall in the past year (OR 2.10, 95\% CI: 1.24-3.58) and occasionally/often report feeling of losing balance (OR 2.12, 95\% CI: 1.17-3.84). Conversely, women were 65\% less likely than men to consume three alcoholic drinks or more per day (OR 0.35, 95\% CI: 0.20-0.61). Increasing age was significantly associated with increasing number of fall risk factors in the univariate analysis. However, no association was found between age and number of risk factors in the multivariable analysis.

Conclusions This is the first study to explore fall risk factors among middle-aged adults presenting to ED using self-reported data. Our findings present valuable information that is critical for informing evidence-based strategies for falls prevention policy and initiatives which aim to reduce the burden of disease in this population in later life.

\section{DIFFERENTIAL TRENDS IN FALL-RELATED FRACTURE AND NON-FRACTURE HOSPITALISATIONS FOR PEOPLE WITH DEMENTIA}

1,2Lara Harvey, ${ }^{3}$ Rebecca Mitchell, ${ }^{2,4}$ Brian Draper, ${ }^{2,4}$ Henry Brodaty, ${ }^{1,2}$ Jacqueline Close. ${ }^{1}$ Neuroscience Research Australia, Australia; ${ }^{2}$ University of New South Wales, Australia; ${ }^{3}$ Macquarie University, Australia; ${ }^{4}$ Dementia Collaborative Research Centre, Australia

10.1136/injuryprev-2016-042156.95

Background Injury, predominantly fall-related injury, is the most common reason for hospitalisation for people with dementia. Trends in fall-related injury hospitalisations for older people generally have changed over the past decade. It is unknown what impact dementia has on these trends.

Methods Fall-related injury hospitalisations during 1 January 2003 to 31 December 2012 for people aged 65 and older admitted to a hospital in New South Wales, Australia were identified. Hospitalisation records were probabilistically linked to provide comprehensive person-based records. Rates were age-standardised to the 2001 Australian Standard population. Trends over time were analysed using negative binomial regression analysis Results There were 52,502 hospitalisations for people with dementia and 203,330 for people without dementia. People with dementia were more likely to be admitted for a hip fracture
(ARR 1.76; 95\% CI: 1.73-1.79, $\mathrm{p}<0.0001$ ) and traumatic brain injury (TBI) (ARR 1.08; 95\% CI: 1.03-1.14, p = 0.0027), but less likely to be admitted for other (non-hip) fractures (ARR 0.72 ; 95\% CI: $0.71-0.73, \mathrm{p}<0.0001$ ) or non-fracture injuries (ARR 0.96; 95\% CI: 0.95-0.97, p < 0.001). Hospitalisation rates for people with dementia decreased by $4.2 \%$ (95\% CI: $-5.6-2.7, \mathrm{p}<0.001)$ per annum for hip fractures and $1.6 \%$ (95\% CI: $2.3-0.8, \mathrm{p}<0.001)$ per annum for other fractures, but increased by 7.5\% (95\% CI: 4.2-10.8\%, p < 0.0001) for TBI and 2.0\% (95\% CI: $0.1-4.0, \mathrm{p}=0.0388)$ for other non-fracture injuries. In contrast, hip fracture hospitalisation rates remained constant and other fracture and non-fracture injuries increased for people without dementia.

Conclusions Fall-related fracture rates, notably hip fractures, have decreased over the past ten years in people with dementia, whilst there has not been a corresponding decrease in people without dementia. Rates for non-fracture injuries including TBI have increased in both people with and without dementia. The reasons for these differences are not clear.

\section{HIP FRACTURE AND THE INFLUENCE OF DEMENTIA ON HEALTH OUTCOMES AND ACCESS TO REHABILIATION FOR OLDER PEOPLE}

1,2 Rebecca Mitchell, ${ }^{2}$ Lara Harvey, ${ }^{3,4}$ Henry Brodaty, 3,4 Brian Draper, 2,4Jacqui Close. ${ }^{1}$ Macquarie University, Australia; ${ }^{2}$ Neuroscience Research Australia, Australia; ${ }^{3}$ Dementia Collaborative Research Centre, Australia; ${ }^{4}$ University of New South Wales, Australia

\subsection{6/injuryprev-2016-042156.96}

Background Recovery following hip fracture can be aided by access to, and participation in, rehabilitation-related activities. However, access to rehabilitation can differ for individuals with and without dementia. This study compares the characteristics and health outcomes of individuals with and without dementia following a hip fracture; and their access to and outcomes following hospital-based rehabilitation.

Methods An examination of hip fractures involving individuals aged 65 years and older with and without dementia using linked hospital separation, rehabilitation and mortality records during 1 January 2009 to 31 December 2013 in New South Wales, Australia. Comorbidities were identified using a 1-year lookback period and a modified Charlson Comorbidity Index. Logistic regression was used to examine the association of a hospitalbased rehabilitation and individual characteristics.

Results There were 8,785 individuals with and 23,520 individuals without dementia who sustained a hip fracture. Individuals with dementia had a higher age-adjusted 30-day mortality rate compared to individuals without dementia (11.7\% vs $5.7 \%)$, a lower proportion of age-adjusted 28 -day re-admission $(17.3 \%$ vs $24.4 \%$ ), and a longer age-adjusted mean length of stay (22.2 vs 21.9 days). Compared to individuals without dementia, individuals with dementia had 4.3 times (95\% CI: 3.90-4.78) lower odds of receiving hospital-based rehabilitation. However, when they did receive rehabilitation they achieved significant motor functional gain at discharge compared to admission assessed using the Functional Independence Measure $(p<0.0001)$, but to a lesser extent than individuals without dementia.

Conclusions Within a population-based cohort, older individuals with dementia can benefit from access to, and participation in, rehabilitation activities following a hip fracture. This will ensure that they have the best chance of returning to their pre-fracture physical function and mobility. 